\title{
Lower extremity kinematics during forward heel-slip
}

\author{
Sukwon Kim ${ }^{\mathrm{a}}$, Kyung-Sook Joo ${ }^{\mathrm{b}}$, Jian $\mathrm{Liu}^{\mathrm{c}}$ and Jee-Hoon Sohn ${ }^{\mathrm{d}, *}$ \\ ${ }^{a}$ Department of Physical Education, College of Education, Chonbuk National University, Jeonju, Korea \\ ${ }^{\mathrm{b}}$ Department of Nursing Science, U1 University, Yeongdong-gun, Chungcheongbuk-do, Korea \\ ${ }^{\mathrm{c}}$ Division of Safety Technology, Marshall University, Huntington, WV, USA \\ ${ }^{\mathrm{d}}$ Department of Physical Education, College of Cultural Convergence, Jeonju University, Jeonju, Korea
}

\begin{abstract}
.
BACKGROUND: Most fall intervention studies attempted to improve the mobility, range of motion of upper and lower extremities, or all major muscle strengths. Yet, there has been little effort to identify movements or actions that may be mainly responsible for recovering from a slipping. It was imperative to link lower extremity kinematics in conjunction with the functional anatomy of lower extremity muscles during forward heel-slipping to identify what muscles should have been activated substantially if a person would have recovered from forward heel-slipping.

OBJECTIVE: The present study investigated lower extremity movements, such as the ankle, knee, and hip rotations, which could contribute to falls from forward heel-slipping. Determining changes in positions of foot, shank, and thigh during slipping would provide information to develop the optimal training regimen or interventions that may be effective for improving a chance to recover from the postural disturbance.

METHODS: Twenty healthy adults (24-68 years old) participated in this experiment. Among twenty participants, only eight participants' data were analyzed in this study. The 3D position data were used to compute the sagittal foot, shank, and thigh angles and frontal thigh angle.

RESULTS: The study results indicated that, during the period of slipping, the angles of the segments of the slipping leg were different from that of the foot, shank, and thigh when walking ordinarily over the dry surface in the present study.

CONCLUSIONS: The characteristics or differences in the angular kinematics of lower extremity during unexpected slips in the present study demonstrate possible causes for slip-induced falls.
\end{abstract}

Keywords: Forward heel slip, leg kinematics, segment angles, fall

\section{Introduction}

Slips and falls are a main cause of accidents in the home and workplace accounting for $30 \%$ of all injuries [1,2]. According to the Liberty Mutual Workplace Safety Index, falls on the same level were the major sources of slip and fall accidents leading to time-loss injuries and ranked second with direct cost of $\$ 11.23$ billons accounting for 19.2 percent of the total injury burden [2]. Often, slip or fall accidents result in the bruise, hip or wrist fracture, or head injuries causing permanent physical damages or deaths [3,4]. Despite constant attempts to decrease falls/fall-related injuries, falls and fall-related injuries constantly stay in the leading cause of injury death for the elderly [4-6]. People start exhibiting a

\footnotetext{
${ }^{*}$ Corresponding author: Jee-Hoon Sohn, Department of Physical Education, College of Cultural Convergence, Jeonju University, Jeonju, Korea. Tel.: +82 63220 4667; Fax: +82 63220 2596; E-mail: jhsohn@jj.ac.kr.
}

0928-7329/19/\$35.00 (c) 2019 - IOS Press and the authors. All rights reserved

This article is published online with Open Access and distributed under the terms of the Creative Commons Attribution NonCommercial License (CC BY-NC 4.0). 
tendency of falling with advancing age because an aging body is constantly to lose its ability to integrate neuro-musculo-skeletal systems resulting in an imbalance. Lower body reactions or reactive movements have received much attention since lower extremity was suggested to play a dominant active role in terms of corrective response [5-8]. Although kinematics analyzes movement patterns without the forces that cause the movement, the acquisition of limb kinematics during walking and slipping should provide significant information about limb control and insight into muscle performance.

Most falls related to walking are initially provoked by environmental factors such as the uneven surface, slippery surface, height, slopes, vibration, etc. However, whether a person falls or not depends upon one's physical ability to recover from the initial environmental factors that trigger instability [9-12]. For a person to regain their stability when one loses balance, the person must be able first to detect postural disturbances through the central nervous system or peripheral nervous system [13]. Then, one must be able to produce smooth and coordinated movements across the body joints to overcome the instability and regain balance [5,9-11]. Thus, an intact neuro-musculo-skeletal system is a key factor in producing such movements.

Poor mobility and health status have been suggested as the leading cause for the decrease in physical abilities such as strength attenuation contributing to fall-related accidents among older adults [14] due to impaired postural control $[9,13,15,16]$ or deterioration in motor control $[13,17]$.

Furthermore, many studies $[5,9,13,15,16,18,19]$ proposed that strength training and balance training among older adults developed an improved mobility and health status. Most studies attempted to improve the range of motion of upper and lower extremities and to strengthen all major muscle. Yet, there has been little effort to identify movements or actions that may be mainly responsible for recovering from a slipping.

In the present paper, authors were challenged to investigate lower extremity movements, such as the ankle, knee, and hip rotations, which could contribute to falls from a forward heel-slipping. The study endeavored to link lower extremity kinematics in conjunction with the functional anatomy of lower extremity muscles during forward heel-slipping to identify what muscles should have been activated substantially if a person would have recovered from forward heel-slipping. Determining changes in positions of foot, shank, and thigh during slipping would provide information to develop the optimal training regimen or interventions that may be effective for improving a chance to recover from the postural disturbance. This study further tried to establish proper strategies for trainers as to what muscles should be strengthened to recover from forward hill-slipping.

\section{Method}

\subsection{Subjects}

Sample size calculation [20] was performed and a sample of 8 were sufficient to satisfy Type I error of 0.05 and Type II error of $<0.2$ (Power $>0.8$ ). Eight participants (19-27 years old) participated in this study. In order for experimenters to compare lower extremity kinematics while walking on dry surface and on a contaminated surface, participants who slipped forward more than five $\mathrm{cm}$ during slip trials were drawn for data comparison. The young adults or older adults were respectively recruited from the local university or the local community. Each participant completed an inform consent procedure approved by the University's Institutional Review Board (IRB). Participants were excluded from the study if they indicated any physical problems (i.e. hip, knee, ankle problems). 


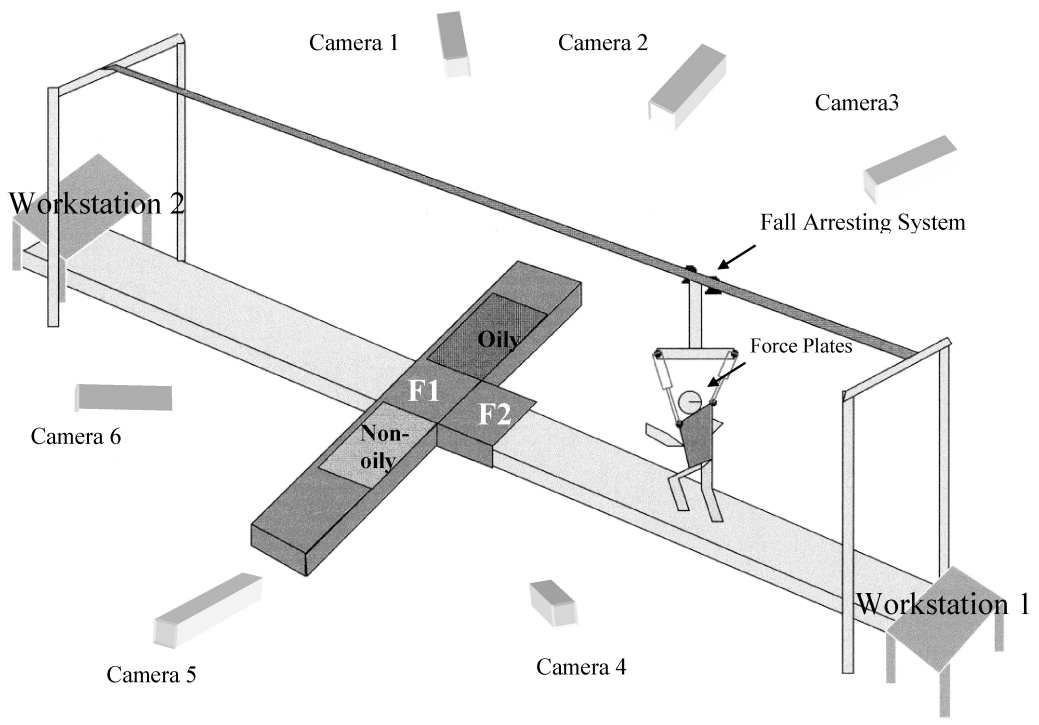

Fig. 1. Field layout of the experiment including Fall Arresting System, Infra-red cameras (6), Linear Slide Floor Changer (LSFC) with force plate (F2) and hidden oily and non-oily test floor surfaces, fixed force plate (F1), and workstations.

\subsection{Apparatus}

A vinyl tile (Armstrong) was installed on the top of a walking track (Fig. 1). To create a realistic slippery environment, the vinyl tile surface was sprayed with a soap and water mixture (2:3) resulting in the dynamic coefficient of friction (COF) of 0.07. The available dynamic COF (ADCOF) of the vinyl surface was measured by a standard $4.54 \mathrm{~kg}(10 \mathrm{lb}$.) horizontal pull slip-meter with a rubber sole material on the floor. An overhead fall arresting harness system was used to prevent fall injuries during walking (Fig. 1). In order to provide unexpected slippery condition, each subject's eyes were always fixed on a TV located $1.5 \mathrm{~m}$ high. A fall-arresting harness was designed to permit a subject's body to drop approximately $15 \mathrm{~cm}$. A six-camera system (Qualysis) collected three-dimensional position data at $120 \mathrm{~Hz}$.

\subsection{Procedure}

A set of 26 markers were placed on the anatomically significant landmarks to present a whole body. Among them, twelve markers such as right and left heels (2), medial malleolus (2), lateral malleolus (2), medial epicondyle (2), lateral epicondyle (2), and ASIS (2) were used for data analysis of the current study. Within a 20-min session, experimenters introduced a slippery surface while the participant's postural data were recorded (Fig. 1). One slip trial was performed for each subject since the subjects would alter their walking characteristics once they were exposed to a slippery surface. That effect did not allow the study to collect multiple slip trials for each subject. To conceal any sound of the floor changing, a Walkman was provided and played old comedy routines.

\subsection{Data analysis and statistics}

The 3D kinematics data from the cameras were collected for calculating the sagittal foot, shank, and thigh angles and frontal thigh angle. The data were recorded for five seconds at $120 \mathrm{~Hz}$ and low-pass filtered $\left(4^{\text {th }}\right.$ order, Butterworth, cutoff $\left.=12 \mathrm{~Hz}\right)$. 
Descriptive and inferential statistical analyses were performed (JMP package, SAS Institute Inc. Cary, NC, USA). One-way ANOVA was used to test the group differences. The results were considered as statistically significant when $p \leqslant 0.05$.

\subsection{Dependent variables}

The definitions of lower extremity segmental orientations in this study were similar to ISB recommendations [21]. Specifically, the segmental coordinate systems were first constructed in the following manner:

For the foot segment, the origin was defined as the inter-malleolar point located midway between markers at medial and lateral malleoli. The $\mathrm{Z}$ axis was defined as a line connecting the heel marker and the origin, and pointing cranially. The $\mathrm{YZ}$ axis was defined as a line connecting the medial and lateral malleolus markers, and pointing to the right. With the Gram-Schmidt orthogonalization process [22], the $\mathrm{X}$ and $\mathrm{Y}$ axes were then determined. The $\mathrm{X}$ axis was a line perpendicular to the plane spanned by $\mathrm{YZ}$ and $\mathrm{Z}$ axes, pointing anteriorly, while the $\mathrm{Y}$ axis was the common line perpendicular to both $\mathrm{X}$ and $\mathrm{Z}$ axes.

For the shank segment, the origin was defined as the same origin of the foot segment on the same side. The $\mathrm{Y}$ axis was defined as a line connecting the medial and lateral condyle markers, and pointing to the right. The $\mathrm{YZ}$ axis was defined as a line connecting the origin and the midpoint between medial and lateral condyle markers, and point cranially. With the orthogonalization process, the $\mathrm{X}$ and $\mathrm{Z}$ axes were determined as the line perpendicular to the plane spanned by $\mathrm{Y}$ and $\mathrm{YZ}$ axes, pointing anteriorly, and the common line perpendicular to both $\mathrm{X}$ and $\mathrm{Y}$ axes, respectively.

For the thigh segment, the origin was coincident with the hip joint, which was defined based on left and right ASIS markers [23]. The $\mathrm{Z}$ axis was defined as a line connecting the origin and the midpoint between medial and lateral condyle markers. The YZ axis was defined as a line connecting medial and lateral condyle markers, and pointing to the right. With the orthogonalization process, the $\mathrm{X}$ and $\mathrm{Y}$ axes were determined as a line perpendicular to the plane spanned by $\mathrm{Z}$ and $\mathrm{YZ}$ axes, pointing anteriorly, and the common line perpendicular to both $\mathrm{X}$ and $\mathrm{Z}$ axes, respectively.

After the segmental coordinate systems were established, the segmental 3D Euler angles were calculated using XYZ convention [23]. The following conventions were exercised: $+\mathrm{X}$ represents abduction and adduction for left and right side, respectively; $+\mathrm{Y}$ represents flexion for both sides; $+\mathrm{Z}$ represents external and internal rotation for left and right side, respectively.

\subsubsection{Sagittal foot, shank, and thigh angle}

Right and left sagittal foot, shank, and thigh angles were assessed to evaluate differences in angle changes (flexion or extension) between walking-on-slippery-surface trials and walking-on-dry-surface trials. Vectors of foot, shank, and thigh were defined as Fig. 1. These vectors and ground X vector were used to calculate angles (Fig. 2). Slip-start (SS) and slip-end (SE) points [24] were identified for data normalization. SS was defined as the point where non-rearward positive acceleration of the heel after heel contact (HC), equivalently where the first minimum of the horizontal heel velocity after HC [8,24]. SE for SDI was defined as the point where peak horizontal heel acceleration occurred after SS point $[8,24]$. The continuous foot, shank, and thigh angles were normalized from HC to SS and from SS to SE [8,24]. First, every angle at 20, 40, 60, 80, 100\% of the first normalized phase (HC to SS) was evaluated. Second, every angle at 20,40,60,80,100\% of the second normalized phase (SS to SE) was compared between normal walking data and slipping data. 


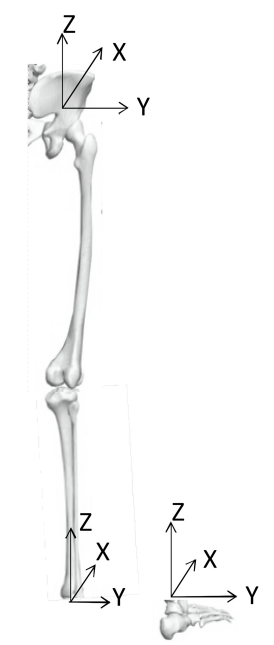

Fig. 2. Vectors of foot, shank, and thigh.

\subsubsection{Frontal thigh angle}

Frontal thigh angle was calculated to determine if thigh angle deteriorates in the frontal plane; frontal thigh deteriorations assisted to identify rotation of the thigh segment during events. Transverse thigh angle was not assessed since there was almost no rotation occurred at longitudinal axis. Ground $\mathrm{Z}$ vector and thigh were used to calculate the frontal thigh angle while slipping or walking. As same as sagittal angle, SS and SE points were identified for data normalization [24]. The continuous frontal thigh angles were normalized from HC to SS and from SS to SE. Since there was no difference between the dry and slippery surface, up to $50 \%$ of a gait cycle, basic information was statistically assessed at 10, 30, 50\%. Afterward, information was statistically assessed at every $10 \%$. Every angle at 10, 30, 50, 70, 90\% of the first normalized phase (HC to SS) and the second normalized phase (SS to SE) was evaluated while slipping and walking.

\section{Results}

While slipping, sagittal left foot angle continuously became smaller until SE. This reduction in foot angle indicated that, while slipping, the supporting leg's knee joint dropped close to the floor surface quickly. The supporting foot keeps rotating forward after SS compared to normal walking. Such forward rotating foot angle was found to be significantly lower during slipping than during normal walking (Table 1). It indicates that instead of completing the swing phase, the supporting foot rapidly rotates to bring the toe down to the ground, which is a typical toe-touch strategy to establish a wider base-ofsupport. Overall, while slipping, the slipping leg's sagittal foot angle became smaller (Figs 3 and 4). The sagittal angle of the slipping foot after SS was significantly lower during slippery trials than during normal walking. It indicates that the slipping foot reached foot flat condition faster while slipping. The sagittal angle of the supporting shank keeps rotating forward even after SS during the slipping trials (Fig. 5). Together with the increasing sagittal thigh angle, the knee joint of the supporting side was found to flex more during slipping compared to that during normal walking (Table 1). Both the thigh segments on both supporting side and slipping side adducted more during slipping than during normal walking (Table 1). 
Table 1

Descriptive statistics of segment angles during gait cycle and $\mathrm{HC}$ to SE

\begin{tabular}{|c|c|c|c|}
\hline \multirow[t]{2}{*}{ Dependent variables } & \multicolumn{2}{|c|}{ Mean (standard deviation) } & \multirow[t]{2}{*}{$p$-value } \\
\hline & Dry & Slippery & \\
\hline \multicolumn{4}{|l|}{ Sagittal left foot angles } \\
\hline $10 \%$ gait cycle & $174.31(3.32)$ & $174.34(4.48)$ & 0.99 \\
\hline $30 \%$ gait cycle & $165.23(2.60)$ & $165.09(2.67)$ & 0.91 \\
\hline $50 \%$ gait cycle & $159.60(3.32)$ & $159.35(4.45)$ & 0.90 \\
\hline $60 \%$ gait cycle & $145.81(7.18)$ & $145.91(7.94)$ & 0.97 \\
\hline $70 \%$ gait cycle & $106.65(13.68)$ & $107.27(11.90)$ & 0.92 \\
\hline $80 \%$ gait cycle & $102.58(7.02)$ & $102.60(6.77)$ & 0.99 \\
\hline $90 \%$ gait cycle & $134.31(8.93)$ & 130.15 (18.64) & 0.58 \\
\hline $10 \%$ HC-SS & $182.08(3.74)$ & $182.09(5.30)$ & 0.99 \\
\hline $30 \%$ HC-SS & $166.38(2.31)$ & $166.43(2.48)$ & 0.97 \\
\hline $50 \%$ HC-SS & $165.31(2.56)$ & $165.16(2.62)$ & 0.91 \\
\hline $70 \%$ HC-SS & $163.05(2.48)$ & $163.00(2.71)$ & 0.97 \\
\hline $90 \%$ HC-SS & $157.77(3.66)$ & $157.91(4.45)$ & 0.95 \\
\hline $10 \%$ SS-SE & $148.29(7.06)$ & $146.23(8.06)$ & 0.60 \\
\hline $30 \%$ SS-SE & 147.09 (7.49) & $135.80(12.82)$ & $0.05^{*}$ \\
\hline $50 \%$ SS-SE & $145.81(7.92)$ & $121.87(19.31)$ & $0.006^{*}$ \\
\hline $70 \%$ SS-SE & $144.46(8.36)$ & $108.56(24.22)$ & $0.001^{*}$ \\
\hline $90 \%$ SS-SE & $143.02(8.81)$ & $105.04(20.90)$ & $0.0003^{*}$ \\
\hline \multicolumn{4}{|l|}{ Sagittal right foot angles } \\
\hline $10 \%$ gait cycle & $138.56(8.47)$ & $137.08(10.34)$ & 0.76 \\
\hline $30 \%$ gait cycle & $107.69(3.77)$ & $109.56(4.53)$ & 0.38 \\
\hline $50 \%$ gait cycle & $183.77(5.17)$ & $184.50(4.09)$ & 0.76 \\
\hline $60 \%$ gait cycle & $179.56(5.16)$ & $180.37(5.48)$ & 0.76 \\
\hline $70 \%$ gait cycle & $165.88(1.60)$ & $169.97(5.50)$ & $0.05^{*}$ \\
\hline $80 \%$ gait cycle & $164.86(1.67)$ & $171.85(6.60)$ & $0.01^{*}$ \\
\hline $90 \%$ gait cycle & $163.35(1.85)$ & $169.85(6.94)$ & $0.02^{*}$ \\
\hline $10 \%$ HC-SS & $147.17(7.04)$ & $145.90(8.21)$ & 0.74 \\
\hline $30 \%$ HC-SS & $102.50(11.10)$ & $102.92(12.18)$ & 0.94 \\
\hline $50 \%$ HC-SS & $106.60(3.53)$ & $108.07(5.34)$ & 0.52 \\
\hline 70\% HC-SS & $147.97(5.06)$ & $149.43(6.24)$ & 0.61 \\
\hline $90 \%$ HC-SS & $189.46(5.56)$ & $190.03(4.00)$ & 0.81 \\
\hline $10 \%$ SS-SE & $183.41(4.88)$ & $181.16(4.67)$ & 0.36 \\
\hline $30 \%$ SS-SE & $181.81(4.64)$ & $174.33(5.57)$ & $0.01^{*}$ \\
\hline $50 \%$ SS-SE & $180.21(4.42)$ & $172.25(5.92)$ & $0.009^{*}$ \\
\hline $70 \%$ SS-SE & $178.64(4.23)$ & $172.49(6.66)$ & $0.04^{*}$ \\
\hline $90 \%$ SS-SE & $177.08(4.07)$ & $172.64(7.16)$ & 0.15 \\
\hline \multicolumn{4}{|c|}{ Sagittal left shank angle (degree) } \\
\hline $10 \%$ gait cycle & $105.64(2.47)$ & $105.24(2.13)$ & 0.74 \\
\hline $30 \%$ gait cycle & $86.60(4.07)$ & $86.179(4.22)$ & 0.84 \\
\hline $50 \%$ gait cycle & $73.54(2.70)$ & $73.62(2.92)$ & 0.95 \\
\hline $60 \%$ gait cycle & $59.92(4.46)$ & $60.23(3.81)$ & 0.88 \\
\hline $70 \%$ gait cycle & $41.91(5.89)$ & $41.68(4.30)$ & 0.92 \\
\hline $80 \%$ gait cycle & $38.04(7.02)$ & $35.46(3.20)$ & 0.15 \\
\hline $90 \%$ gait cycle & $60.59(5.63)$ & $46.56(11.96)$ & $0.001^{*}$ \\
\hline $10 \%$ HC-SS & $108.88(2.31)$ & $108.52(1.60)$ & 0.72 \\
\hline $30 \%$ HC-SS & $93.80(1.27)$ & $93.76(4.38)$ & 0.98 \\
\hline $50 \%$ HC-SS & $86.82(4.23)$ & $86.36(4.22)$ & 0.83 \\
\hline $70 \%$ HC-SS & $80.50(2.94)$ & $80.63(3.21)$ & 0.93 \\
\hline $90 \%$ HC-SS & 70.99 (3.27) & $80.63(0.13)$ & 0.81 \\
\hline $10 \%$ SS-SE & $61.91(4.78)$ & $60.56(3.94)$ & 0.54 \\
\hline $30 \%$ SS-SE & $61.04(4.85)$ & $54.75(5.48)$ & $0.03^{*}$ \\
\hline $50 \%$ SS-SE & $60.16(4.92)$ & $48.30(7.93)$ & $0.003^{*}$ \\
\hline $70 \%$ SS-SE & 59.27 (4.99) & $42.87(9.33)$ & $0.0006^{*}$ \\
\hline $90 \%$ SS-SE & $58.36(5.06)$ & $39.95(8.99)$ & $0.0002^{*}$ \\
\hline
\end{tabular}


Table 1, continued

\begin{tabular}{|c|c|c|c|}
\hline \multirow[t]{2}{*}{ Dependent variables } & \multicolumn{2}{|c|}{ Mean (standard deviation) } & \multirow[t]{2}{*}{$p$-value } \\
\hline & Dry & Slippery & \\
\hline \multicolumn{4}{|c|}{ Sagittal right shank angle (degree) } \\
\hline $10 \%$ gait cycle & $55.74(4.40)$ & $55.37(5.44)$ & 0.88 \\
\hline $30 \%$ gait cycle & $42.67(3.09)$ & $43.25(4.54)$ & 0.77 \\
\hline $50 \%$ gait cycle & $109.31(5.50)$ & $109.64(5.04)$ & 0.90 \\
\hline $60 \%$ gait cycle & $108.63(3.35)$ & $108.64(2.54)$ & 0.99 \\
\hline $70 \%$ gait cycle & $93.65(3.66)$ & $101.90(5.71)$ & $0.004^{*}$ \\
\hline $80 \%$ gait cycle & $86.50(3.40)$ & $106.73(8.93)$ & $<0.0001^{*}$ \\
\hline $90 \%$ gait cycle & $82.45(2.48)$ & $98.93(11.64)$ & $0.001^{*}$ \\
\hline $10 \%$ HC-SS & $60.91(4.06)$ & $60.47(4.98)$ & 0.85 \\
\hline $30 \%$ HC-SS & $40.00(4.86)$ & $40.38(5.66)$ & 0.88 \\
\hline $50 \%$ HC-SS & $42.00(3.06)$ & $42.32(4.36)$ & 0.86 \\
\hline $70 \%$ HC-SS & $74.13(5.11)$ & $74.70(6.24)$ & 0.84 \\
\hline $90 \%$ HC-SS & $114.26(4.22)$ & $114.26(3.28)$ & 0.99 \\
\hline $10 \%$ SS-SE & $110.29(3.61)$ & $108.91(2.71)$ & 0.40 \\
\hline $30 \%$ SS-SE & $109.54(3.53)$ & $105.77(3.42)$ & $0.04^{*}$ \\
\hline $50 \%$ SS-SE & $108.83(3.48)$ & $103.97(4.44)$ & $0.03^{*}$ \\
\hline $70 \%$ SS-SE & $108.14(3.46)$ & $104.48(5.30)$ & 0.12 \\
\hline $90 \%$ SS-SE & $107.48(3.46)$ & $105.92(4.95)$ & 0.48 \\
\hline \multicolumn{4}{|c|}{ Sagittal left thigh angle (degree) } \\
\hline $10 \%$ gait cycle & $109.59(4.05)$ & $109.94(5.84)$ & 0.89 \\
\hline $30 \%$ gait cycle & $94.16(5.28)$ & $94.18(5.35)$ & 0.99 \\
\hline $50 \%$ gait cycle & $77.86(5.04)$ & $77.89(4.32)$ & 0.99 \\
\hline $60 \%$ gait cycle & $74.85(4.30)$ & $74.87(3.67)$ & 0.99 \\
\hline $70 \%$ gait cycle & $83.27(1.63)$ & $83.33(3.00)$ & 0.96 \\
\hline $80 \%$ gait cycle & $101.04(1.30)$ & $100.48(4.16)$ & 0.72 \\
\hline $90 \%$ gait cycle & $112.47(2.40)$ & $104.24(6.88)$ & $0.006^{*}$ \\
\hline $10 \%$ HC-SS & $110.60(3.55)$ & $110.94(4.96)$ & 0.87 \\
\hline $30 \%$ HC-SS & $106.04(4.52)$ & $106.27(6.90)$ & 0.94 \\
\hline $50 \%$ HC-SS & $94.39(5.22)$ & $94.71(5.16)$ & 0.90 \\
\hline $70 \%$ HC-SS & $84.66(4.84)$ & $84.62(4.48)$ & 0.98 \\
\hline $90 \%$ HC-SS & $76.61(4.96)$ & $76.73(4.06)$ & 0.96 \\
\hline $10 \%$ SS-SE & $74.91(4.42)$ & $74.92(3.69)$ & 0.99 \\
\hline $30 \%$ SS-SE & $74.90(4.35)$ & $76.13(3.50)$ & 0.54 \\
\hline $50 \%$ SS-SE & $74.92(4.20)$ & $79.54(4.51)$ & $0.05^{*}$ \\
\hline $70 \%$ SS-SE & $74.98(4.20)$ & $84.70(7.36)$ & $0.006^{*}$ \\
\hline $90 \%$ SS-SE & $75.06(4.12)$ & $90.13(10.13)$ & $0.002^{*}$ \\
\hline \multicolumn{4}{|c|}{ Sagittal right thigh angle (degree) } \\
\hline $10 \%$ gait cycle & $74.29(4.32)$ & 74.46 (4.49) & 0.94 \\
\hline $30 \%$ gait cycle & $105.25(4.02)$ & $104.37(3.87)$ & 0.66 \\
\hline $50 \%$ gait cycle & $109.94(4.19)$ & $109.78(4.57)$ & 0.91 \\
\hline $60 \%$ gait cycle & $109.32(4.62)$ & $108.99(4.65)$ & 0.89 \\
\hline $70 \%$ gait cycle & $106.67(5.87)$ & $105.84(5.88)$ & 0.78 \\
\hline $80 \%$ gait cycle & $97.18(6.04)$ & $105.17(5.49)$ & $0.01^{*}$ \\
\hline $90 \%$ gait cycle & $87.03(5.51)$ & $117.19(10.12)$ & $<0.0001^{*}$ \\
\hline $10 \%$ HC-SS & $73.57(4.09)$ & $73.58(5.51)$ & 0.99 \\
\hline $30 \%$ HC-SS & $84.52(4.86)$ & $84.42(5.51)$ & 0.97 \\
\hline $50 \%$ HC-SS & $104.67(4.21)$ & $103.73(4.19)$ & 0.66 \\
\hline 70\% HC-SS & $114.25(3.66)$ & $113.43(3.50)$ & 0.65 \\
\hline $90 \%$ HC-SS & $108.60(3.84)$ & $108.52(4.22)$ & 0.97 \\
\hline $10 \%$ SS-SE & $109.25(4.37)$ & $108.99(4.41)$ & 0.90 \\
\hline $30 \%$ SS-SE & $109.32(4.41)$ & $108.01(5.01)$ & 0.58 \\
\hline $50 \%$ SS-SE & $109.34(4.46)$ & $106.77(5.40)$ & 0.32 \\
\hline $70 \%$ SS-SE & $109.31(4.51)$ & $106.77(5.40)$ & 0.17 \\
\hline $90 \%$ SS-SE & $109.26(4.59)$ & $105.29(6.03)$ & 0.16 \\
\hline
\end{tabular}


Table 1, continued

\begin{tabular}{|c|c|c|c|}
\hline \multirow[t]{2}{*}{ Dependent variables } & \multicolumn{2}{|c|}{ Mean (standard deviation) } & \multirow[t]{2}{*}{$p$-value } \\
\hline & Dry & Slippery & \\
\hline \multicolumn{4}{|c|}{ Frontal left thigh angle (degree) } \\
\hline $10 \%$ gait cycle & $96.80(2.26)$ & $95.94(1.70)$ & 0.40 \\
\hline $30 \%$ gait cycle & $91.67(2.37)$ & $91.27(2.85)$ & 0.76 \\
\hline $50 \%$ gait cycle & $94.12(1.53)$ & $93.84(1.86)$ & 0.75 \\
\hline $60 \%$ gait cycle & $96.37(1.40)$ & $96.34(1.82)$ & 0.97 \\
\hline $70 \%$ gait cycle & $96.89(1.31)$ & $96.52(2.34)$ & 0.70 \\
\hline $80 \%$ gait cycle & $96.86(1.21)$ & $93.74(2.90)$ & $0.01^{*}$ \\
\hline $90 \%$ gait cycle & $96.99(1.31)$ & $91.70(2.58)$ & $0.0001^{*}$ \\
\hline $10 \%$ HC-SS & $97.30(2.05)$ & $96.65(2.51)$ & 0.47 \\
\hline $30 \%$ HC-SS & $93.47(2.32)$ & $92.86(2.51)$ & 0.62 \\
\hline $50 \%$ HC-SS & $91.77(2.32)$ & $91.20(2.82)$ & 0.66 \\
\hline 70\% HC-SS & $92.85(2.47)$ & $92.61(2.53)$ & 0.85 \\
\hline 90\% HC-SS & $94.52(1.69)$ & $94.17(1.77)$ & 0.69 \\
\hline $10 \%$ SS-SE & $95.99(1.68)$ & $96.27(1.91)$ & 0.76 \\
\hline $30 \%$ SS-SE & $96.13(1.62)$ & $96.66(2.16)$ & 0.59 \\
\hline $50 \%$ SS-SE & $96.28(1.56)$ & $96.64(2.32)$ & 0.72 \\
\hline $70 \%$ SS-SE & $96.40(1.51)$ & $96.27(2.35)$ & 0.90 \\
\hline $90 \%$ SS-SE & $96.50(1.46)$ & $95.44(2.39)$ & 0.30 \\
\hline \multicolumn{4}{|c|}{ Frontal right thigh angle (degree) } \\
\hline $10 \%$ gait cycle & $79.18(4.94)$ & $79.32(5.15)$ & 0.96 \\
\hline $30 \%$ gait cycle & $81.34(4.07)$ & $81.21(4.13)$ & 0.95 \\
\hline $50 \%$ gait cycle & $81.43(4.02)$ & $81.03(4.54)$ & 0.86 \\
\hline $60 \%$ gait cycle & $82.09(3.85)$ & $82.00(5.07)$ & 0.97 \\
\hline $70 \%$ gait cycle & $83.42(4.11)$ & $83.65(5.41)$ & 0.93 \\
\hline $80 \%$ gait cycle & $82.46(4.04)$ & $83.64(5.25)$ & 0.62 \\
\hline $90 \%$ gait cycle & $80.54(4.56)$ & $84.62(7.10)$ & 0.20 \\
\hline $10 \%$ HC-SS & $78.51(4.86)$ & $78.65(5.00)$ & 0.95 \\
\hline $30 \%$ HC-SS & $81.18(4.66)$ & $80.92(5.07)$ & 0.91 \\
\hline $50 \%$ HC-SS & $81.30(4.14)$ & $81.17(4.10)$ & 0.95 \\
\hline $70 \%$ HC-SS & $81.62(4.28)$ & $81.27(4.19)$ & 0.87 \\
\hline 90\% HC-SS & $81.65(4.01)$ & $81.17(4.48)$ & 0.82 \\
\hline $10 \%$ SS-SE & $81.86(3.80)$ & $81.17(4.48)$ & 0.97 \\
\hline $30 \%$ SS-SE & $81.92(3.81)$ & $82.65(5.49)$ & 0.76 \\
\hline $50 \%$ SS-SE & $81.99(3.82)$ & $83.26(5.79)$ & 0.61 \\
\hline $70 \%$ SS-SE & $82.07(3.83)$ & $83.49(5.71)$ & 0.57 \\
\hline 90\% SS-SE & $82.16(3.85)$ & $83.42(5.43)$ & 0.60 \\
\hline
\end{tabular}

Mean (SD) of foot, shank, and thigh angles during walking on dry surface or slippery surface. HC (Heel Contact), SS (Slip-Start), SE (Slip-End). For example, $10 \%$ HC-SS indicates during a period from heel contact to slipstart. ${ }^{*} p$-value $<0.05$.

\section{Discussion}

The objective of the current study was to compare the lower extremity angular kinematics during unexpected forward heel-slipping with that during normal dry surface walking. As hypothesized, statistically significant floor surface condition effect was found between the two different surfaces.

In the results from the present study, authors were challenged to link lower extremity rotational kinematics in conjunction with functional anatomy of lower extremity muscles. This association between the two would assist trainers to identify specific muscles that should be trained in order for a person to recover from a postural disturbance during slipping. The results found in the present study, such as relatively smaller left and right foot angles, smaller left and right shank angles, and larger left thigh angles 


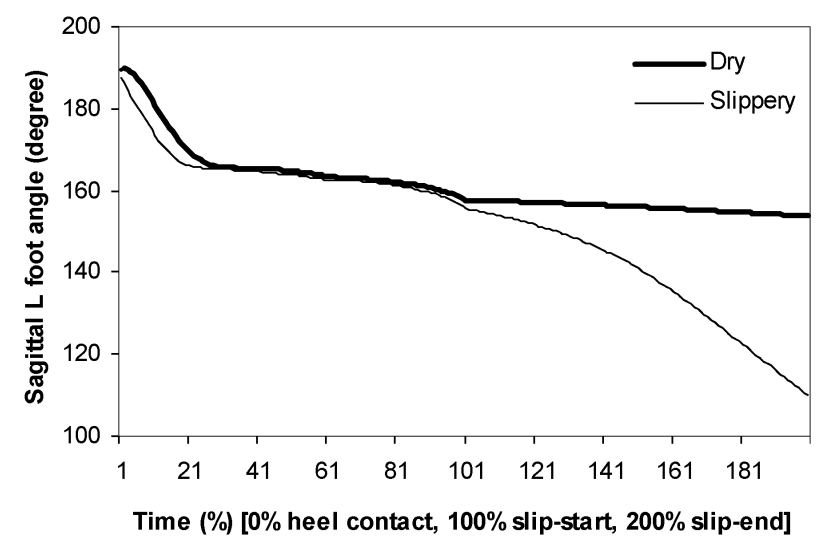

Fig. 3. Sagittal left foot (supporting foot) angle.

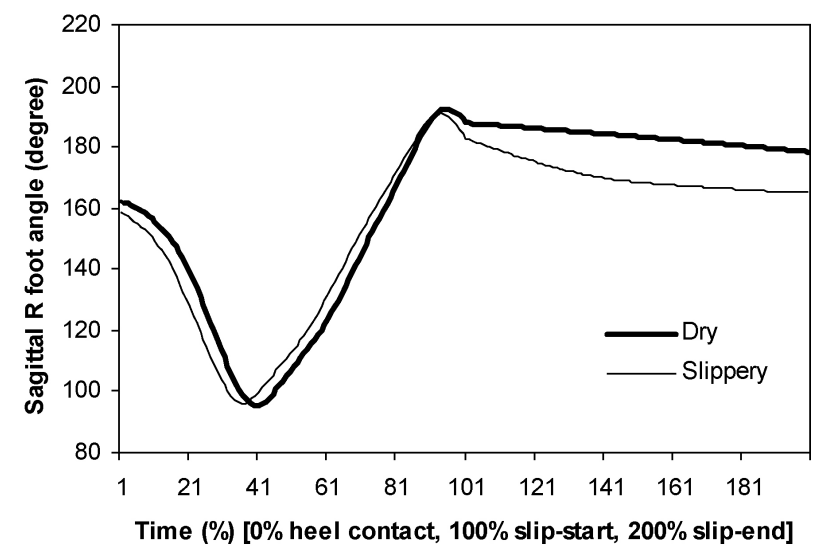

Fig. 4. Sagittal right foot (slipping foot) angle.

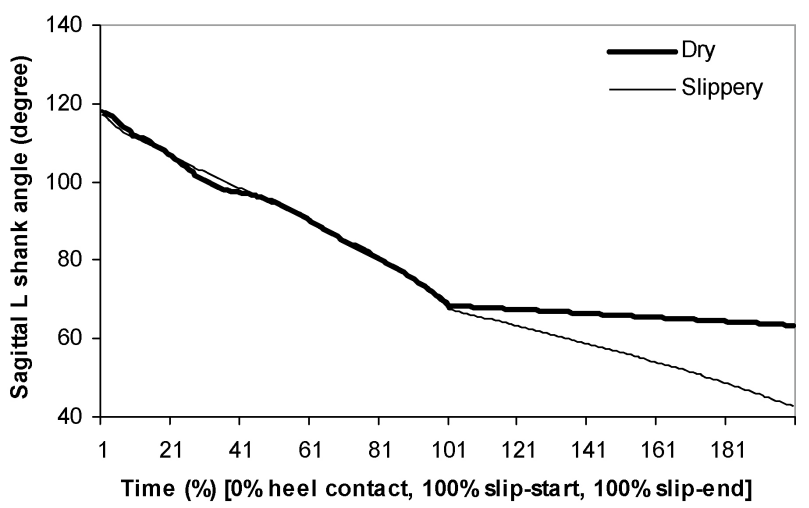

Fig. 5. Sagittal left shank angle.

during a period from heel-contact to slip-end, may have suggested that knee extensor muscles, ankle plantar flexion muscles, and hip extensor muscles could be functionally involved in recovering from postural disturbances after a slip would start. In order to actively recover from a slipping, knee extensor 
muscles such as vastus lateralis, medialis, and intermedius and rectus femoris should actively contract to stabilize the knee joint of the support leg and ankle plantar flexion muscles such as gastronemius, soleus, and Plantaris muscles. Proper strengthening of any of these muscles could improve one's ability to plantar-flex the foot increasing the chance to recover from a slip. In addition, hip extensor muscles, such as hamstring muscles (biceps femoris, semimembranosus, semitendinosus), gluteus maximus and medius, and adductor magnus, should actively contract to stability hip joint of the slipping leg.

Overall, the study results revealed the role of the unperturbed limb in response to slip perturbations. After slip starts, the unperturbed limb (non-dominant) demonstrated a strategy quickly to land on the floor surface with decreased sagittal foot angle and shank angle, and increased sagittal thigh angle. This finding is consistent with literatures [25-28]. Employing the landing strategy, the subjects could quickly establish a stable base-of-support with the slipping limb. A controlled base-of-support was important for subjects to regain balance after postural perturbation such as a slipping. Martelli et al. [28] suggested that the unperturbed limb should be better suited for keeping balance in response to perturbations. The reactive control of stability of the unperturbed limb had an impact on recovery of the whole body because the non-dominant limb preferentially provided support to propulsion provided by the dominant limb [28,29]. The results from the present study also agreed to that the reactive joint motions of the unperturbed limb during slipping were essential for maintaining balance or stability or supporting body weight. Similar kinematic pattern was observed for the perturbed limb while slipping in the present study. Towards the end of a slip, the perturbed limb was characterized by a decreasing sagittal foot angle, shank angle, and thigh angle (not statistically significant), the supporting limb was characterized by increasing sagittal thigh angle. Together, such characteristic was consistent with the effort to maintain body center-of-mass over the perturbed limb while slipping. Apparently, lower extremity functional capability (e.g., joint torque production) could play an important role in the success of such strategy [14-16]. Again, proper strengthening program would be essential in improving the chance of recovery during a slipping or a postural disturbance.

Moderate reductions in falls risk (15-20\%) were found in healthy older adults after exercise intervention such as strength, balance, or aerobic exercise [30,31]. However, general exercise interventions for falls risk reduction demonstrated the lack of effectiveness and consistency for some special population [32-34]. A possible cause of these results was alleged to be the lack of task specificity to the recovery actions during a postural disturbance [35,36]. Like results shown in the present study, to counteract to the change in lower extremity movements during slipping, rotations of lower extremity segments should be actively executed [37,38]. It was suggested that interventions that stimulate such recovery movements or mechanisms should be more effective than general exercise interventions [39-41]. Accordingly, task-specific training such as perturbation-based balance training (PBT) has been shown to improve reactive balance control after postural disturbances in some laboratory studies $[15,18,40-43]$. These studies [15,18,40-43] proposed that older adults' reactive locomotor adaptation potential could be augmented by employing task specific stimuli such as PBT.

As a pilot study, the current research had several limitations that should be addressed in the future. Due to limited sample size, the study results should be generalized with caution. Walking over a slippery surface typically would result in either a balance recovery or a fall. But, the slip outcome of each perturbation trial was not considered in the current study. Future studies are needed to pinpoint the relationship between lower extremity angular kinematics with slip outcomes. Potential interaction effect with aging or clinical populations could also greatly expand one's understanding of slip-induced fall mechanism. 


\section{Conclusion}

In conclusion, the characteristics or differences in the angular kinematics of lower extremity during unexpected slips in the present study demonstrate possible causes for slip-induced falls and appear to be a practical approach to relate exercise intervention to reduce a slip-induced fall accident. Based on the current evidence, it appears that task specific training interventions such as PBT might be the most effective methods in improving reactive balance control through active control of muscle contraction at the joints in realistic situations. To better determine the linkage between the functional anatomy and rotational kinematics, more controlled studies of lower extremity kinematics during postural disturbance should be required.

\section{Acknowledgments}

The authors would like to thank Dr. Thurmon Lockhart for his insightful guidance in developing the current research area.

\section{Conflict of interest}

The authors declare that they have no competing interests.

\section{References}

[1] The Liberty Mutual Workplace Safety Index, 2016

[2] The Liberty Mututal Workplace Safety Index, 2018.

[3] Sterling DA, O'Connor JA, Bonadies J. Geriatric falls: injury severity is high and disproportionate to mechanism. J Trauma. 2001; 50: 116-19.

[4] Burns ER, Stevens JA, Lee R. The direct costs of fatal and non-fatal falls among older adults - United States. J Safety Res. 2016; 58: 99-103.

[5] Sohn J, Park S, Kim S. Effects of dancesport on walking balance and standing balance among the elderly. Technology and Health Care. 2018; 26: S481-S490.

[6] Liu J, Lockhart, TE. Comparison of 3D joint moments using local and global inverse dynamics approaches among three different age groups. Gait \& Posture. 2006; 23(4): 480-485.

[7] Grundstrom AC, Guse CE, Layde PM. Risk factors for falls and fall-related injuries in adults 85 years of age and older. Arch Gerontol Geriatr. 2012; 54(3): 421-428.

[8] Kim SW, Lockhart TE, Yoon HY. Relationship between walking velocity and slip-induced fall accidents. Safety Science. 2005; 43: 425-436.

[9] Kim S, Lockhart T. Lower limb control and mobility following exercise training. Journal of NeuroEngineering and Rehabilitation. 2012; 9: 15. doi: 10.1186/1743-0003-9-15.

[10] Yang F, Bhatt T, Pai Y. Limits of recovery against slip-induced falls while walking. Journal of Biomechanics. 2011; 44(15): 2607-2613.

[11] Hu X, Qu X. Differentiating slip-induced falls from normal walking and successful recovery after slips using kinematic measures. Ergonomics. 2013; 56(5): 856-867.

[12] Bohm S, Mademli L, Mersmann F, Arampatzis A. Predictive and reactive locomotor adaptability in healthy elderly: A systematic review and meta-analysis. Sports Med. 2015; 45(12): 1759-1777.

[13] Sohn J, Kim S. Falls study: proprioception, postural stability, and slips. Bio-Medical Materials and Engineering. 2015; 26: S693-S703.

[14] Cho KH, Bok SK, Kim YJ, Hwang SL. Effect of lower limb strength on falls and balance of the elderly. Ann Rehabil Med. 2012; 36(3): 386-393.

[15] Gerards M, McCrum C, Mansfield A, Meijer K. Perturbation-based balance training for falls reduction among older adults: current evidence and implications for clinical practice. 2017; 17(12): 2294-2303. 
[16] Grabiner MD, Crenshaw JR, Hurt CP, Rosenblatt NJ, Troy KL. Exercise-based fall prevention: Can you be a bit more specific? Exerc Sport Sci Rev. 2014; 42(4): 161-168.

[17] Woollacott M, Shumway-Cook A. Attention and the control of posture and gait: a review of an emerging area of research. Gait and Posture. 2002; 16: 1-14.

[18] Mansfield A, Peters AL, Liu BA, Maki BE. Effect of a perturbation-based balance training program on compensatory stepping and grasping reactions in older adults: A randomized controlled trial. Phys Ther. 2010; 90(4): 476-491.

[19] Seo J, Kim S. Prevention of potential falls of elderly healthy women: gait asymmetry. Educational Gerontology. 40(2): 123-137.

[20] DSS RESEARCH. Calculator, Retrieved from https://www.dssresearch.com/KnowledgeCenter/toolkitcalculators/sampl esizecalculators.aspx.

[21] Wu G, Siegler S, Allard P, Kirtley C, Leardini A, Rosenbaum D, Whittle M, D’Lima D, Cristofolini L, Witte H, Schmid $\mathrm{O}$, Stokes I. ISB recommendation on definition of joint coordinate system of various joint for the reporting of human joint motion-part I: ankle, hip, and spine. J of Biomechanics. 2002; 35(4): 543-548.

[22] Liu J, Lockhart TE. Comparison of 3D joint moments using local and global inverse dynamics approaches among three different age groups. Gait \& Posture. 2006; 23(4): 480-485.

[23] Bell AL, Pedersen DR, Brand RA. A comparison of the accuracy of several hip center location prediction methods. Journal of Biomechanics. 1990; 23(6): 617-621.

[24] Lockhart TE, Woldstad JC, Smith JL. Effects of age-related gait changes on the biomechanics of slips and falls. Ergonomics. 2003; 46: 1136-1160.

[25] Marigold DS, Bethune AJ, Patla AE. Role of the unperturbed limb and arms in the reactive recovery response to an unexpected slip during locomotion. Journal of Neurophysiology. 2003; 89(4): 1727-1737.

[26] Yang F, Anderson FC, Pai YC. Predicted threshold against backward balance loss following a slip in gait. Journal of Biomechanics. 2008; 41: 1823-1831.

[27] Allen JL, Kautz SA, Neptune RR. Step length asymmetry is representative of compensatory mechanisms used in poststroke hemiparetic walking. Gait Posture. 2011; 33: 538-543.

[28] Martelli D, Monaco V, Bassi Luciani L, Micera S. Angular momentum during unexpected multidirectional perturbations delivered while walking. IEEE Trans. Biomed. Eng. 2013; 60: 1785-1795.

[29] Sadeghi H, Allard P, Duhaime M. Functional gait asymmetry in able-bodied subjects. Hum. Mov. Sci. 1997; 16: $243-$ 258.

[30] Gillespie LD, Robertson MC, Gillespie WJ, et al. Interventions for preventing falls in older people living in the community. Cochrane Database Syst Rev. 2012; 9: CD007146.

[31] Sherrington C, Whitney JC, Lord SR, Herbert RD, Cumming RG, Close JC. Effective exercise for the prevention of falls: A systematic review and meta-analysis. J Am Geriatr Soc. 2008; 56(12): 2234-2243.

[32] Canning CG, Sherrington C, Lord SR, et al. Exercise for falls prevention in Parkinson disease: A randomized controlled trial. Neurology. 2015; 84(3): 304-312.

[33] Allen NE, Canning CG, Sherrington C, et al. The effects of an exercise program on fall risk factors in people with Parkinson's disease: A randomized controlled trial. Mov Disord. 2010; 25(9): 1217-1225.

[34] Verheyden GS, Weerdesteyn V, Pickering RM, et al. Interventions for preventing falls in people after stroke. Cochrane Database Syst Rev. 2013; 5: CD008728.

[35] Oddsson LIE, Boissy P, Melzer I. How to improve gait and balance function in elderly individuals - compliance with principles of training. Eur Rev Aging Phys Act. 2007; 4(1): 15-23.

[36] Grabiner MD, Crenshaw JR, Hurt CP, Rosenblatt NJ, Troy KL. Exercise-based fall prevention: Can you be a bit more specific? Exerc Sport Sci Rev. 2014; 42(4): 161-168.

[37] Hof AL. The equations of motion for a standing human reveal three mechanisms for balance. J Biomech. 2007; 40(2): 451-457.

[38] Maki BE, McIlroy WE. Change-in-support balance reactions in older persons: An emerging research area of clinical importance. Neurol Clin. 2005; 23(3): 751-783.

[39] Grabiner MD, Crenshaw JR, Hurt CP, Rosenblatt NJ, Troy KL. Exercise-based fall prevention: Can you be a bit more specific? Exerc Sport Sci Rev. 2014; 42(4): 161-168.

[40] Mansfield A, Peters AL, Liu BA, Maki BE. Effect of a perturbation-based balance training program on compensatory stepping and grasping reactions in older adults: A randomized controlled trial. Phys Ther. 2010; 90(4): 476-491.

[41] Carty CP, Cronin NJ, Nicholson D, et al. Reactive stepping behaviour in response to forward loss of balance predicts future falls in community-dwelling older adults. Age Ageing. 2015; 44(1): 109-115.

[42] Pai YC, Bhatt TS. Repeated-slip training: An emerging paradigm for prevention of slip-related falls mong older adults. Phys Ther. 2007; 87(11): 1478-1491.

[43] Pai YC, Bhatt T, Wang E, Espy D, Pavol MJ. Inoculation against falls: Rapid adaptation by young and older adults to slips during daily activities. Arch Phys Med Rehabil. 2010; 91(3): 452-459. 